\title{
Etude de la décontamination électrocinétique de l'aluminium d'une boue de laiterie
}

\author{
Belgacem Mansouri ", Sabir Hazourli ", Isabelle Le Hecho ${ }^{b}$, Sylvaine Tellier ${ }^{b}$ \\ a : Laboratoire de traitement des eaux et valorisation des déchets industriels, \\ département de chimie, université de Badji-Mokhtar, BP 12, Annaba 23000 (Algérie) \\ $\mathrm{b}$ : Laboratoire de chimie analytique bio inorganique et environnement (LCABIE), \\ UMR CNRS 5034, université de Pau et des Pays de L'Adour, France - Téléphone :05 59407739 -Télécopie :05 59407674
}

Pour toute correspondance : bel_mansouri@yahoo.fr -Tél/fax:00 21338876567

\begin{abstract}
Résumé
Le succès du procédé de décontamination électrocinétique des métaux d'une boue dépend du choix des conditions expérimentales : courant ou tension appliqué, $\mathrm{pH}$, réactifs ajoutés, débit du flux électro-osmotique, etc. Des conditions bien adaptées doivent permettre notamment de minimiser les phénomènes d'adsorption, d'augmenter la solubilité pour éviter la précipitation du métal dans la boue. Ces précautions, entre autres, facilitent la récupération cathodique du métal. Dans cette étude de décontamination électrocinétique de l'aluminium d'une boue provenant d'eaux résiduaires de laiterie, il a été décidé de garder un débit électro-osmotique et un potentiel constants successivement à $10 \mathrm{ml} / \mathrm{h}$ et $15 \mathrm{~V}(\mathrm{IV} / \mathrm{cm})$, et de faire circuler dans le réacteur un milieu basique $(\mathrm{NaOH} 0,1 \mathrm{M})$ des deux côtés des compartiments anodique et cathodique. Ce choix de pH extrême a permis d'évaluer l'électromigration de l'espèce complexe mononucléaire de l'aluminium et non de l'aluminium monomère $\left(\mathrm{Al}^{3+}\right)$ qui est largement prédominant en milieu acide $(\mathrm{pH}=4,6)$. Les résultats obtenus ont montré l'efficacité de cette technique d'électromigration pour récupérer l'aluminium complexe $\mathrm{Al}(\mathrm{OH})_{5}{ }^{2-}$. En effet, à $\mathrm{pH}$ supérieur à 10 , mesuré dans les compartiments électrolytiques, l'aluminium redevient soluble et la récupération de ce métal atteint $72 \%$.
\end{abstract}

Mots clés : aluminium, boue, caractérisation, décontamination, électromigration, laiterie.

\section{Introduction}

Les essais de caractérisation des eaux résiduaires d'une laiterie industrielle fabricant du lait reconstitué pasteurisé et du fromage à pâte molle de type « camembert » ont montré une forte et irrégulière charge polluante. Le prétraitement de ces eaux résiduaires par coagulation floculation au sulfate d'aluminium réduit considérablement la charge organique : la $\mathrm{DBO}_{5}$ et la $\mathrm{DCO}$ subissent un abattement de l'ordre de $99 \%$ avec une turbidité finale de l'eau prétraitée comparable à celle de l'eau potable (Hazourli et al., 2007).
Ce prétraitement appliqué aux eaux urbaines ou industrielles a pour inconvénient majeur de générer des volumes importants de boue qui peuvent contenir des métaux lourds et d'autres composés toxiques (Ayoub et Merhebi, 2002). Par contre, pour les effluents de laiterie, même si le volume formé est important ( $200 \mathrm{ml} / \mathrm{l}$ d'eau traitée), soit la boue est dépourvue de substances toxiques, soit ces dernières sont présentes à des teneurs inférieures aux limites de la norme Afnor U44-04 I (Moletta et Torrijos, 1999). Toutefois, pour plus de sécurité, la boue doit faire l'objet d'un traitement approprié dans le cas d'une valorisation agricole, avicole ou autre. Plusieurs méthodes de dépollution des boues sont déjà testées ; elles sont chimiques, thermiques, physiques, etc. Ces méthodes demandent en général des moyens techniques et financiers importants (Koller, 2004).

Dans cette étude, nous examinons un procédé d'extraction électrocinétique a priori peu coûteux, l'électrodépolIution ou électroremédiation, qui utilise un courant électrique circulant entre deux électrodes inattaquables. Différents phénomènes physiques peuvent être générés par ces systèmes électrodes/sol ou boue : l'électroosmose, l'electrophorèse, l'electromigration et les phénomènes chimiques liés aux réactions électrochimiques prés des électrodes comme l'électrolyse de l'eau (Acar et al., 1995 ; Acar et Alshawabkeh, 1996). Beaucoup de travaux de laboratoire utilisant cette technique sont cités dans la littérature, après les premiers essais de Lageman et al., 1989. Très peu d'essais au stade pilote et sur le terrain ont été appliqués en Europe et aux Etats-Unis (Tellier et Astruc, 2000). II a été démontré une efficacité intéressante pour une grande variété d'espèces chimiques : les métaux lourds, qui ont été largement étudiés (Lake, 1987), ainsi que les composés organiques (Lageman et al., 2005). L'objectif de cette étude est orienté vers l'application de cette technique électrocinétique pour la décontamination de la boue de laiterie de l'aluminium utilisé comme coagulant lors de la clarification des eaux résiduaires.

La mise en évidence de la toxicité de l'aluminium est récente, la forme monomère étant la plus toxique pour I'homme et son environnement (Guibaud et Ayele, 1997). 
En consommation d'eau potable, l'aluminium a été limité en France à $200 \mu \mathrm{g} / \mathrm{l}$ (décret du 3 janvier 1989). Après une caractérisation nécessaire de la boue, toutes les conditions analytiques de minéralisation et d'extraction de l'aluminium de cette boue ainsi que les conditions expérimentales de l'étude électrocinétique ont été fixées de telle manière à obtenir un transfert maximum de ce métal de l'anode à la cathode.

\section{Matériels et méthodes}

\section{Récupération et méthodologie d'analyse de la bove}

L'eau résiduaire prélevée subit aussitôt un traitement de clarification physico-chimique par coagulation-floculation suivant le protocole expérimental du jar-test (Boeglin, 1999). Dans tous les essais de clarification, $400 \mathrm{mg} / \mathrm{l}$ de coagulant (sulfate d'alumine) et I ppm de floculant (polyélectrolyte) sont employés. Après 30 minutes de décantation, la clarification de l'eau est maximale (turbidité résiduelle 8 NTU). Le volume de boue liquide obtenu après ce temps de décantation est compris entre 200 à $250 \mathrm{ml} / \mathrm{l}$ d'eau résiduaire (Hazourli et al., 2007). La boue contient la fraction particulaire et colloïdale décantable enlevée à l'eau résiduaire laitière, notamment la matière organique, le phosphore mais aussi une quantité de réactifs chimiques (précipités des coagulants, de réactifs de désinfection) ainsi qu'une flore bactérienne banale voire pathogène. Étant donné la biodégradabilité rapide de la boue obtenue, tous les paramètres susceptibles d'être altérés ont été analysés dans les plus brefs délais en accord avec les règles de conservation et méthodes d'analyses normalisées (AFEE, 1985). Toutes les mesures ont été reproduites sur au moins trois échantillons identiques de boue liquide ou sèche. Le pH (appareil portable C535), la conductivité (appareil Mettler Toledo MC226), les chlorures et les sulfates sont mesurés dans le surnageant de la boue liquide centrifugée pendant I heure avec les mêmes modes opératoires normalisés utilisés pour l'analyse des eaux résiduaires (Hazourli et al., 2007). Les matières sèches (MS) sont mesurées par différence de masse après une dessiccation de $10 \mathrm{~g}$ de boue liquide à $100-105{ }^{\circ} \mathrm{C}$ pendant 24 h. Le résidu calciné à $525 \pm 25{ }^{\circ} \mathrm{C}$ pendant 3 heures (ou matières volatiles sèches, MVS) permet de distinguer les matières organiques des matières minérales. La matière organique (MO) s'obtient par la différence entre ces résidus secs et calcinés ; néanmoins, la quantité de $\mathrm{MO}$ calculée sera approximative. La mesure de la matière grasse dans la boue liquide a été réalisée par la méthode de Gerber (Salgarolo, 1990). L'azote et le phosphore total ont été analysés sur $10 \mathrm{~g}$ de boue liquide selon les méthodes décrites par la norme française (AFEE, 1985). L'aluminium, le fer, le sodium, le potassium, le cuivre, le magnésium et le cuivre ont d'abord été extraits de 200 $\mathrm{mg}$ de boue sèche par minéralisation oxydante $(10 \mathrm{ml}$ de $\mathrm{HNO}_{3}$ à $65 \%$ et $5 \mathrm{ml}$ de $\mathrm{H}_{2} \mathrm{O}_{2}$ à $20 \%$ ) sur micro-ondes (Ethos I Millestone).
Le minéralisât récupéré subit une filtration à $0,45 \mu \mathrm{m}$ puis ajusté à $50 \mathrm{ml}$ à l'eau ultra pure de 18,2 Mø.cm.

Ces éléments minéralisés ont été analysés par la torche à plasma à émission atomique (ICPAES-Model Panorama 6I) pour l'aluminium, le fer, le calcium, le magnésium et le cuivre alors que le sodium et le potassium ont été mesurés par la spectrophotométrie de flamme (appareil Corning type 410). L'analyse microbiologique de la boue a ciblé les coliformes totaux, fécaux et les streptocoques fécaux en suivant les protocoles expérimentaux de Lassee, 1984.

\section{Équipement et conditions opératoires}

L'équipement présenté dans cette étude (figure I) a fait ses preuves notamment dans la décontamination des métaux lourds sur des sols modèles (Le Hécho et al., 1998) et sur des boues d'épuration urbaines (Larranaga, 1996). Par ailleurs, un essai a été réalisé au stade pilote dans le cas d'une pollution fluorée et a montré que l'extrapolation des résultats obtenus au laboratoire se faisait de façon satisfaisante (Costarramone et al., 1998).

L'expérience de dépollution de la boue de laiterie envisagée a eu lieu dans une cellule acrylique parallélépipédique à trois compartiments : anodique, cathodique et central. Ce dernier compartiment est limité aux extrémités par un filtre en tissu polyamide afin de faciliter le transport ionique sans que les particules de boue ne passent dans les compartiments électrolytiques.

Dans ce compartiment central de dimension $10 \times 4 \times 4$ $\mathrm{cm}$, il a été introduit une masse de $83 \mathrm{~g}$ de boue sèche mélangée préalablement à $122 \mathrm{ml}$ de $\mathrm{NaOH}$ à 0,001 $\mathrm{mol} / \mathrm{l}$. Ce réactif est injecté en continu dans les compartiments anodique et cathodique à l'aide de deux pompes péristaltiques (Gilson M 312, France) à raison de 0,1 mol/l à $10 \mathrm{ml} / \mathrm{h}$. Cet électrolyte permet de maintenir un $\mathrm{pH}$ basique stable dans toute la cellule. Dans les compartiments électrolytiques de volume $40 \mathrm{ml}$ chacun, est placé un fil de platine comme électrode. Les volumes de solution évacués des trop-pleins sont rigoureusement notés dans le temps afin de calculer les débits électro-osmotiques et les quantités d'aluminium extraites dans chacun des compartiments anodique et cathodique. Une tension continue de $15 \mathrm{~V}$ (gradient de tension égal à $1 \mathrm{~V} / \mathrm{cm}$ ) est appliquée entre les deux électrodes en platine, ce qui correspond à une densité moyenne de courant de 0,637 $\mathrm{A} / \mathrm{cm}^{2}$.

Les principaux paramètres contrôlés dans les compartiments anodique, cathodique et central sont : le courant, la tension, les débits des réactifs et le $\mathrm{pH}$. En fin de manipulation, le lit de boue a été coupé en 5 échantillons de 2 $\mathrm{cm}$ chacun pour analyser l'aluminium. 
Figure 1 : Réacteur électrocinétique utilisé

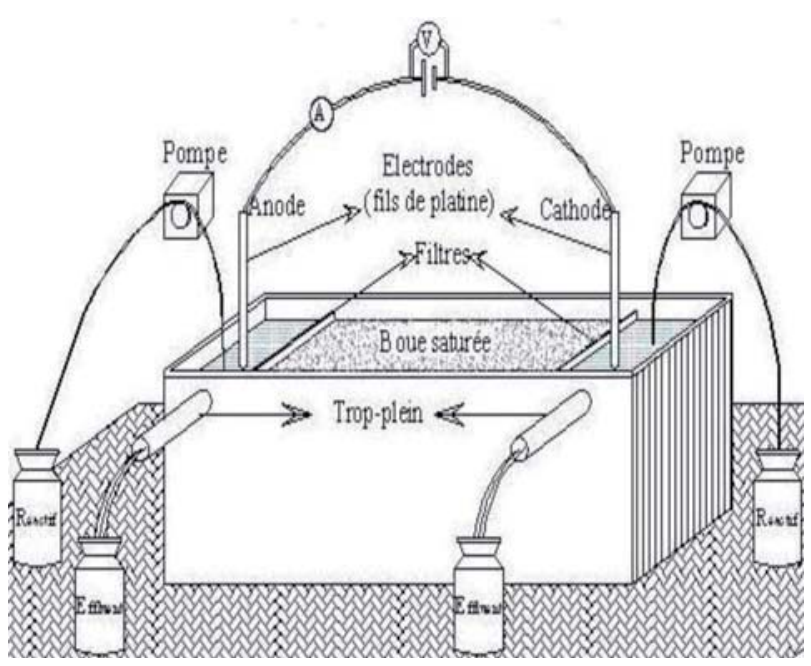

\section{Résultats et discussion}

\section{Caractérisation de la boue}

Les résultats de caractérisation de la boue reportés dans le tableau I montrent sa richesse en éléments minéraux et organiques. La présence d'azote, de phosphore, de potassium et d'alcalino-terreux prédisposerait la boue liquide à être utilisée en amendement des sols. Cependant, la présence de coliformes fécaux et de streptocoques fécaux concentrés dans cette boue limite son épandage dans les sols, d'où la nécessité de l'affiner. A cet égard, il faut noter l'heureuse incidence du chaulage de l'effluent traité, qui entraîne une élévation du $\mathrm{pH}$ et par conséquent un arrêt de la croissance bactérienne et une inhibition du pouvoir fermentescible des boues (Guettier et al., 1994).

D'autre part, l'importante teneur observée en aluminium peut avoir un impact environnemental négatif. Heil et Barbarick, 1989, ont montré que la fixation du phosphore disponible pour les plantes peut être inhibée si les teneurs en sulfate d'aluminium sont supérieures à $10 \mathrm{mg} / \mathrm{g}$. Les teneurs en sulfates et chlorures sont faibles pour être considérées et discutées, par contre la teneur en matière grasse de $100 \mathrm{mg} / \mathrm{l}$ reste par exemple supérieure à la norme algérienne de rejet aqueux industriel $(20 \mathrm{mg} / \mathrm{l})$.

La récupération directe de cette matière grasse de l'eau résiduaire est préférable à l'extraction de la boue, en évitant des déperditions par adsorption de globules gras sur et dans les flocs de boue formés.

Toutes ces analyses de caractérisation présentent une moyenne d'erreur d'environ $\pm 10 \%$.

\section{Tableau 1 : Caractérisation de la bove formée après coagulation}

Température $\left({ }^{\circ} \mathrm{C}\right): 21$

$\mathrm{pH}: 5,4$

Conductivité ( $\mu \mathrm{s} / \mathrm{cm})$ : 1003

MG (mg/L boue liquide) : 100

Chlorures (mg/L boue liquide) : 53

Sulfates (mg/L boue liquide) : 105

Azote total ( $\mathrm{mg} / \mathrm{L}$ boue liquide) : 185

Phosphore ( $\mathrm{mg} / \mathrm{L}$ boue liquide) : 25

MS (mg/L boue liquide) : 2500

$\mathrm{MO}$ (mg/L boue liquide) : 1700

Siccité (\%) : ।,5

MV (\%) : | 4,2

Aluminium(mg Al/g boue sèche) : 16,0

Fer (mg Fe/g boue sèche) : 0, 17

Sodium ( $\mathrm{mg} \mathrm{Na/g}$ boue sèche) : 0,57

Potassium (mg K/g boue sèche) : 0,4I

Calcium (mg Ca/g boue sèche) : I,36

Magnésium(mg Mg/g boue sèche) : 0, 10

Coliformes totaux germe/l00 ml : ।,55

Coliformes fécaux (germe/l00 ml boue liquide) : 128

Streptocoques fécaux (germe/l00 ml boue liquide) : I5

\section{Électromigration de l'aluminium}

\section{Évolution du courant}

Dès le début de l'essai électrocinétique, le courant mesuré aux bornes du réacteur atteint un pic de $25 \mathrm{~mA}$, pour se stabiliser après 12 jours à des valeurs inférieures à $5 \mathrm{~mA}$ (figure 2). Le potentiel imposé à I $5 \mathrm{~V}$ est contrôlé constant durant tout cet essai. Ce pic de courant serait attribué à la dissolution des sels associés initialement à la boue, augmentant ainsi la force ionique et la conductivité dans les trois compartiments de la cellule. La conductivité de la boue mesurée avant l'application de potentiel est déjà élevée $(1003 \mu \mathrm{S} / \mathrm{cm})$. La diminution du courant au cours du temps résulterait de la diminution de la conductivité : les anions et les cations migrent vers l'électrode correspondante et sont neutralisées. Les produits d'électrolyse de l'eau $\left(\mathrm{H}^{+}\right.$et $\mathrm{OH}^{\text {? }}$ à l'anode et à la cathode, respectivement) permettent la neutralisation des espèces ioniques de charges opposées. Cette évolution du courant a été observée par différents auteurs (Le Hécho et al., 1998 ; Costarramone et al., 1998 ; Saichek et Reddy 2003). 
Figure 2 : Évolution du courant entre anode et cathode

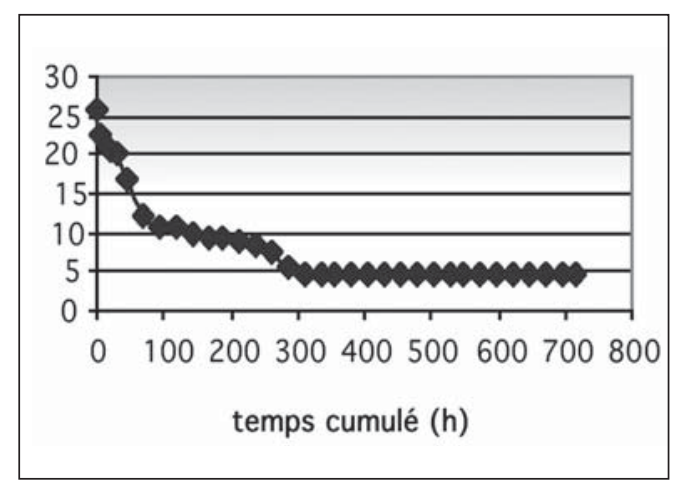

\section{Évolutions du pH}

Dès l'application du potentiel au réacteur, l'électrolyse de l'eau prend place pour générer une oxydation à l'anode avec une production d'ions hydrogène $\left(\mathrm{H}^{+}\right)$et libération d'oxygène gazeux (équation I). A la cathode on assiste à une réduction avec une production d'ions hydroxyl $\left(\mathrm{OH}^{-}\right)$ et libération d'hydrogène gazeux (équation 2). Le pH est alors acide du côté anodique et basique du côté cathodique.

$$
\begin{aligned}
& 2 \mathrm{H}_{2} \mathrm{O} \longrightarrow \mathrm{O}_{2}{ }^{\nearrow}+4 \mathrm{H}^{+}+4 \mathrm{e}^{-} \text {(1) } \\
& 2 \mathrm{H}_{2} \mathrm{O}+2 \mathrm{e}^{-} \longrightarrow \mathrm{H}_{2}+2 \mathrm{OH}^{-} \text {(2) }
\end{aligned}
$$

Les $\mathrm{pH}$ extrêmes aux électrodes créent dans le compartiment central un front acide qui se déplace vers la cathode et un front basique qui se déplace vers l'anode. Le mélange préalable de la boue avec de la soude diluée $(0,00$ I M) et l'ajout en continu de ce réactif plus concentré $(0,0$ I M) aux électrodes permettent d'assurer le passage du courant mais également de neutraliser ces deux fronts acido-basiques et de stabiliser le $\mathrm{pH}$ dans la cellule. On observe (figure 3) que malgré l'ajout de soude, les 3/5 de fractions de la boue sont à un $\mathrm{pH}$ acide $(\sim 6)$ durant la durée de l'expérience. La quatrième fraction dépasse ce $\mathrm{pH}$ à partir du $10^{\mathrm{e}}$ jour, alors que la cinquième à partir $\mathrm{du}$ 4e jour. Les $\mathrm{pH}$ aux électrodes restent basiques (figure 4). Ce front acide ne persistant qu'au niveau du compartiment central est lié probablement à l'origine laitière de la boue. En effet, cette boue utilisée dans des conditions choisies de non chaulage possède nécessairement une biomasse bactérienne considérable (tableau I). La présence de nutriments, le temps long de l'essai, la température à $20^{\circ} \mathrm{C}$ ne font qu'accroître cette population bactérienne qui produit habituellement une acidité lactique. Le mécanisme d'acidification est très connu : des bactéries lactiques comme les Streptococcus et les Lactobacillus secrètent une enzyme (lactase déshydrogénase) qui va permettre d'hydrolyser et transformer le lactose (principal sucre du lait) en glucose et galactose ; l'ensemble des réactions de la glycolyse permettent de produire l'acide lactique de pKa = 3,8 qui, pour un lait fermenté, donne un $\mathrm{pH} \sim 4,5$. Pour rappel, le $\mathrm{pH}$ de la boue avant l'application du potentiel est de 5,4. Cette acidité lactique, permanente tant que les conditions de croissance bactérienne et de nutriments sont réunies, expliquerait sans doute la pérennité du front acide dans cette partie du réacteur. Cette approche bactérienne de maintien et d'autorégulation du pH a été citée déjà pour des sols (Reddy et al., 2003).

\section{Figure 3 : Évolution du profil de pH dans la boue}

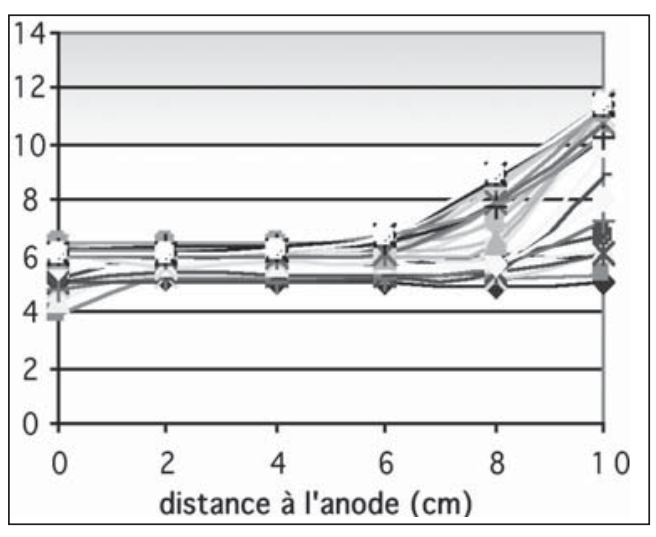

Figure 4 : Evolution du pH à l'anode et à la cathode

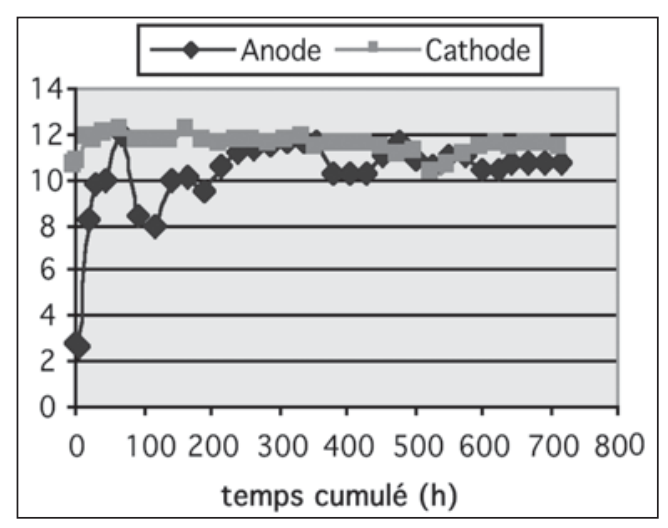

\section{Récupération de l'aluminium de la boue}

Une large part de l'aluminium contenu initialement dans le compartiment central du réacteur ( 328 mg à raison de $16 \mathrm{mg} / \mathrm{g}$ boue sèche) est récupéré sur les électrodes après 30 jours de fonctionnement : I 57 mg à l'anode et 803 mg à la cathode, soit un taux de récupération global proche de 72 \%. (figures 5 et 6). Si l'on rajoute l'aluminium proche de la cathode ( 5 e tranche du réacteur), la récupération avoisine les $77 \%$. Les compartiments anodique, cathodique et la 5 e tranche du compartiment central ayant un $\mathrm{pH}$ supérieur à 10 , la forme prédominante de l'aluminium dans ces milieux est l'ion complexe $\mathrm{Al}(\mathrm{OH})_{5}{ }^{2}$. 
Aux autres $\mathrm{pH}$ mesurés dans les trois premières tranches de la boue ( $\mathrm{pH}$ proche de 6) et dans la $4 \mathrm{e}$ tranche $(\mathrm{pH}$ proche de 8), les espèces prédominantes sont successivement le précipité $\mathrm{Al}(\mathrm{OH})_{3}$ et l'ion $\mathrm{Al}(\mathrm{OH})_{4}$. Pour ces $\mathrm{pH}$, l'espèce $\mathrm{Al}^{3+}$ n'est pas présente, cette espèce se trouvant majoritairement à des $\mathrm{pH}$ inférieurs à 4,6 (Cathalifauld et al., 1997). La bonne récupération de l'aluminium à $\mathrm{pH}$ basique est donc essentiellement due à l'augmentation de la solubilité de ce métal.

L'influence du $\mathrm{pH}$ sur la solubilité de l'aluminium a été vérifiée par une expérience de lixiviation de la boue par différentes solutions aqueuses dans une gamme de $\mathrm{pH}$ allant de I à 12. Les résultats représentés sur la figure 7 montrent que les taux d'extraction les plus importants sont obtenus à $\mathrm{pH}=1$ et $\mathrm{pH}=12$. Beaucoup d'auteurs (Holt et al., 2002 ; Gauthier et Ayele, 2003) rapportent le caractère amphotère de l'aluminium avec une solubilité minimale à $\mathrm{pH}=6,3$.

La spéciation des systèmes à base d'aluminium est très complexe, notamment au niveau des cinétiques de formation des espèces et des mécanismes de leur formation en fonction du pH (Holt et al., 2002). Pour ce qui est du transport électrocinétique de l'aluminium majoritairement de l'anode vers la cathode, sous l'effet du champ électrique, la solubilité de l'aluminium contrôle la mobilité de l'ion complexe. L'électro-osmose peut aussi contribuer à ce transport puisque au niveau de la $4^{\mathrm{e}}$ tranche de boue, il y a confrontation de deux fronts acide et basique, limitant ainsi la mobilité ionique.

Dans ce cas, le transport électro-osmotique pourrait devenir prépondérant. L'application d'un gradient électrique à la boue impliquerait alors les deux mécanismes de transport: l'électro-osmose et l'électromigration. Ces deux mécanismes sont souvent cités dans la décontamination électrocinétique des métaux lourds des sols et des boues (Saichek et Reddy, 2003 ; Reddy et al., 2003 ; Zhongmin et al., 1997).

Figure 5 : Récupération de l'aluminium dans les compartiments anodique et cathodique

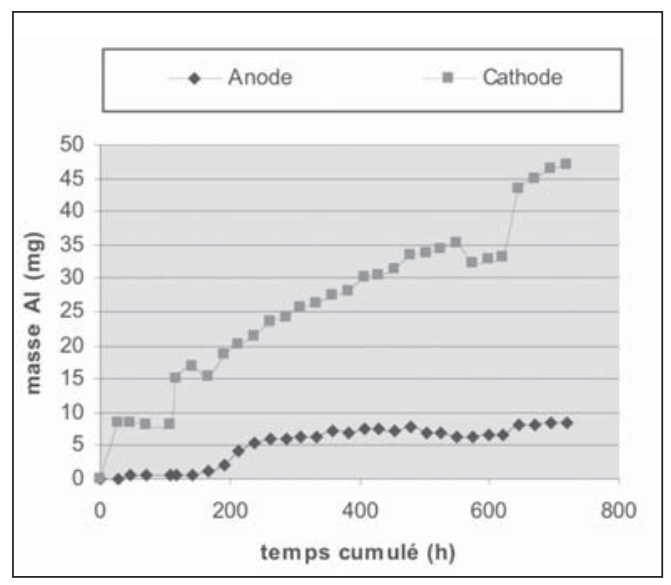

Figure 6 : Récupération de l'aluminium dans les cinq fractions de bove

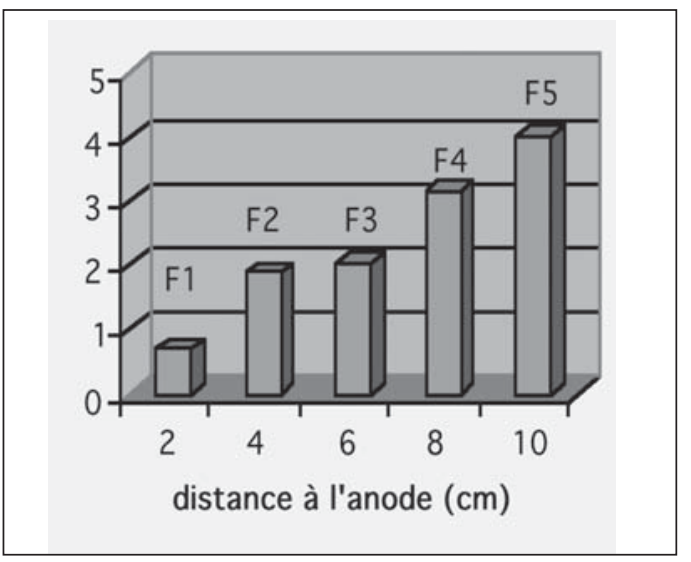

Figure 7 : Variation du taux d'extraction d'aluminium en fonction du $\mathrm{pH}$

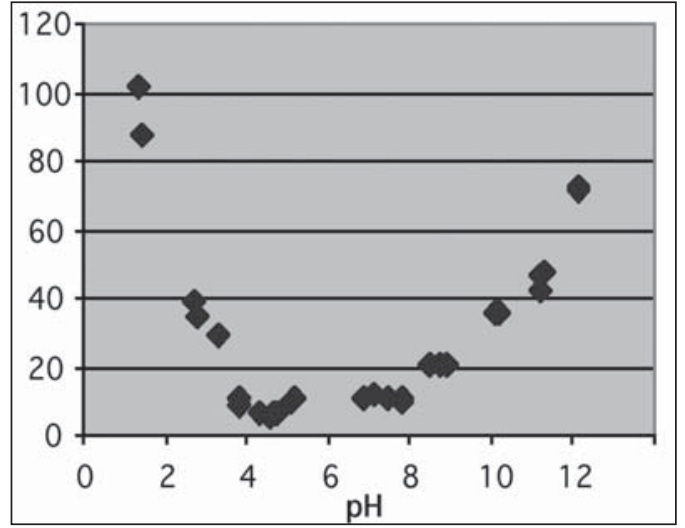

Conclusion

Les résultats obtenus au laboratoire pour l'étude du procédé de décontamination de l'aluminium contenu dans une boue de laiterie sont encourageants : le taux de récupération à la cathode est d'environ $72 \%$. Apparemment, trois formes d'aluminium sont présentes dans les différents compartiments du réacteur, suivant le $\mathrm{pH}: \mathrm{Al}(\mathrm{OH})_{3}(\mathrm{pH} \sim 6), \mathrm{Al}(\mathrm{OH})^{4-}(\mathrm{pH}$ basique $), \mathrm{Al}(\mathrm{OH})_{5}{ }^{2-}$ ( $\mathrm{pH}$ supérieur à 10$)$. Une expérience de lixiviation de la boue pour des $\mathrm{pH}$ variant de I à 12 a confirmé le rôle de la solubilité de l'aluminium sur le transport électrocinétique par électromigration. L'électro-osmose contribuerait à ce transport. 


\section{Références}

Acar Y.B., Gale R.J., Alshawabkeh A.N., Marks R.E., Puppala Bricka S., Parker R.M. (1995), Electrokinetic remediation: basics and technology status, Journal of Hazardous Materials, 40, I17- 137.

Acar Y.B., Alshawabkeh A.N. (1996), Electrokinetic remediation: I. Pilot-scale test with lead-spiked kaolinite, Journal of Geotechnical Engineering , 3, I73- 185.

Ayoub G.M, Merhebi F. (2002), Characteristics and quantities of sludge produced by coagulating wastewater with seawater bittern, lime and caustic, Advances in Environmental Research, 6, 277-284.

Cathalifauld G., Ayele J., Mazet M. (1997), Etude de la complexation des ions aluminium par des molécules organiques : constantes et stœchiométrie des complexes, Water Research, 31, 689-698.

Costarramone N., Tellier S., Grano B., Lecomte D., Astruc M. (1998), Application of an electrokinetic technique to the reclamation of fluoride polluted soils : laboratory and pilot scale experiments, Waste Management Research, 16 , 555-563.

Hazourli S., Boudiba L., Fedaoui D., Ziati M. (2007), Prétraitement par coagulation-floculation d'eaux résiduaires d'une laiterie industrielle, Journal Société Algérienne Chimie, 17, 155-172.

Heil D.M., Barbarick A. (1989), Water treatment sludge influence on the growh of sorghum-sudangrass, Journal of environmental Quality, 18, 292-298.

Holt P.K., Barton G.W., Wark M., Mitchell C.A. (2002), Une comparaison quantitative entre le produit chimique dosant et électrocoagulation, Colloids and Surface, $2 \mathrm{l}$ I, 233-248.

Lageman R., Pool W., Seffinga G. (1989), Electroreclamation: therory and practice, Chemistry Industry, I3, 585-590.

Lageman R., Clarkea R.L., Wiebe P. (2005), Electro-reclamation, a versatile soil remediation solution, Engineering. Geology., 77, |9|-201.

Larranaga J. (1996), Décontamination électrocinétique en métaux lourds appliquée aux boues de station d'épuration urbaine, Thèse de Doctorat n 309, Pau, France, 245 p.

Le Hecho I., Tellier S., Astruc M.(1998), Industrial site soils contaminated with arsenic or chromnium : evaluation of the electrokinetic method, Environmental Technology, 19, |095-| 103.
Reddy K.R., Saichek R.E., Kranti M., Prasanth A. (2002), Effects of soil moisture and heavy metal concentrations on electrokinetic remediation, Indian geotechnical journal, 2, 258-288.

Reddy K.R., Chinthamreddy S., Saichek R.E. (2003), Nutrient Amendment for the Bioremediation of a Chromium-Contaminated Soil by Electrokinetics, Energy Sources, 25, 931-943.

Saichek R.E., Reddy K.R. (2003), Effect of pH control at the anode for the electrokinetic removal of phenanthrene from Kaolin soil, Chemosphere, 5 I, 273-287. 